\title{
Internet of things applied on a supervisory system for modular production system stations
}

\author{
Renan Araújo de Lima ${ }^{1}$, Almir Kimura Junior ${ }^{2}$, Israel Francisco Benítez Pina², Walter Andrés Vermehren \\ Valenzuela $^{2}$ \\ ${ }^{1}$ Universidade do Estado do Amazonas - UEA, Discente do Curso de Eng. de Controle e Automação, Manaus, Amazonas, \\ Brasil. E-mail: <renan.araujo.eng@gmail.com> \\ ${ }^{2}$ Universidade do Estado do Amazonas - UEA, Departamento de Engenharia de Controle e Automação, Mestre em \\ Engenharia Elétrica. E-mail: 〈akimurajr@gmail.com.br> \\ ${ }^{2}$ Universidade do Estado do Amazonas - UEA, Departamento de Engenharia de Controle e Automação, Pós-Doutor em \\ Automação Eletrônica de Processos Elétricos e Industriais. E-mail: 〈benitez.israel@gmail.com.br〉 \\ ${ }^{2}$ Universidade do Estado do Amazonas - UEA, Coordenador do Curso de Eng. de Controle e Automação, Manaus, \\ Amazonas, Brasil, Eng. Indl Mecânico, Doutor em Instrumentação e Controle - UFCG. E-mail: 〈wvalenzuela7@gmail.com>
}

\begin{abstract}
The advent of new technologies gives the companies the possibility to create a strong competitive position by successfully use the recent concepts. New ideals brought the concepts that represents the future fourh industrial revolution, among them is the Internet of Things, which when properly applied, enables systems to have a greater autonomy by the decision taking from data collected from the Internet and the process itself. Considering that most applications of this concept are related to home automation, this article demonstrates the use of Internet of Things in an industrial process represented in laboratory scale, monitored in real time by a supervisory system that initiates production from the demand information simulated by a Web page.
\end{abstract}

Keywords: Internet of Things, Supervisory Systems, Industrial Process.

\section{Sistema supervisório aplicando o conceito de internet das coisas para estações de sistema modular de produção em escala laboratorial}

\section{RESUMO}

O advento de novas tecnologias dá a possibilidade de empresas imporem maior competitividade em relação a outras se os recentes conceitos forem implantados de forma eficiente. Novos ideais deram início aos conceitos que representam a quarta revolução industrial, dentre eles está o conceito de Internet das Coisas, que quando aplicado corretamente, possibilita que sistemas tenham maior autonomia, pela tomada de decisões importantes a partir de dados coletados da Internet e do próprio processo. Tendo em vista que a maioria das aplicações deste conceito estão relacionadas com automação residencial, este artigo demonstra o emprego de Internet das Coisas em um processo industrial representado em escala laboratorial, monitorado em tempo real por um sistema supervisório que inicia a produção a partir da demanda simulada por uma página Web.

Palavras-chaves: Internet das Coisas, Sistemas Supervisórios, Processo Industrial.

\section{INTRODUÇÃO}

A tecnologia está em constante crescimento e cada vez mais apresenta-se no dia a dia do ser humano. Este fato se mostra de grande importância no ramo industrial, pois as empresas devem se adaptar às novidades e implementar os novos conceitos em seus processos para não perder espaço no mercado.

Segundo [1], "O mundo se encontra hoje na transição da terceira para a quarta revolução industrial", implicando que os conceitos que representam a quarta revolução industrial já existem, porém ainda não estão sendo empregados de maneira concisa. Uma das novidades trazidas pelos ideais da nova revolução industrial foi a I.o.T. (Internet of Things), conhecida no Brasil como Internet das Coisas.

A I.o.T. foi definida por [2] como "a aproximação dos objetos físicos do mundo real aos sistemas de informação", que no meio industrial pode ser possível pela interconexão das máquinas e equipamentos do chão de fábrica a uma mesma rede

ITEGAM - JETIA Vol. 01, No. 04. Dezembro de 2015. Manaus - Amazonas, Brasil. ISSN 2447-0228 (ONLINE). 
que também é conectada à Internet. Isso permite que informações importantes que se encontram online, como a demanda de certo produto, possam ser utilizadas para tomadas de decisão direta na produção da fábrica.

O presente artigo pretende implementar um sistema supervisório a um conjunto de estações de sistema modular de produção baseando-se nos pilares que definem o conceito de Internet das Coisas: a conexão de todas as estações a uma mesma rede, conectada também à Internet, de onde serão retiradas as informações de demanda do produto para a tomada de decisão de início de produção; e a utilização de tecnologia RFID, para que todas as informações de produção sejam carregadas em um chip no produto, e posteriormente lidas por cada estação, as quais executarão as ações necessárias de acordo com os dados recebidos.

\section{INTERNET DAS COISAS}

A Internet das Coisas, do inglês Internet of Things, é uma "[...] nova infraestrutura que integra a internet convencional com os objetos permitindo a comunicação entre o mundo virtual e o mundo real" [3]. Seguindo o pensamento de [4], pode-se dizer que é um conceito que considera a presença de coisas/objetos no ambiente, que por meio de conexões com ou sem fio e um esquema de endereçamento único é possível a interação e cooperação com outras coisas/objetos para a criação de novas aplicações ou serviços para atingir um objetivo.

A Internet das Coisas pode ser realizada em três paradigmas: orientado por internet (utilização de dados na internet), orientado por sensores, e orientado por conhecimento [5]. Pode-se dizer que a utilidade real do conceito só será conquistada em aplicações onde os três paradigmas estão inseridos.

A proposta deste artigo procura se utilizar dos três paradigmas: utilizando o dado de demanda obtido de uma página web, utilizando-se da comunicação Ethernet entre os CLPs e o sistema supervisório, possibilitando obter as informações provindas dos sensores das estações de sistema modular de produção, e por último, criando uma tomada de decisão de produção a partir destes dados.

\section{1 PÁGINA WEB}

Um dos pilares do conceito de Internet das Coisas é a orientação por Internet, e a partir desta definição, foi desenvolvida uma página web que simula as compras dos produtos que serão fabricados nas estações de Sistema Modular de Produção.

Quando o número de compras atinge um certo valor, é criado um lote de produção, e a fabricação dos produtos se inicia automaticamente. As informações de cada produto comprado são inseridas em chips RFID que se localizam na matéria prima. Isso permite que ao realizar a leitura dos dados, se saiba quem foi o comprador e qual produto deve ser fabricado.

A página web também permite que se acompanhe em que estágio de produção se encontra o produto que foi comprado, que são: aguardando formação de lote, em fabricação, ou produto finalizado. A seguir pode-se ver a página web desenvolvida para este projeto como mostra a figura 1 e 2 :

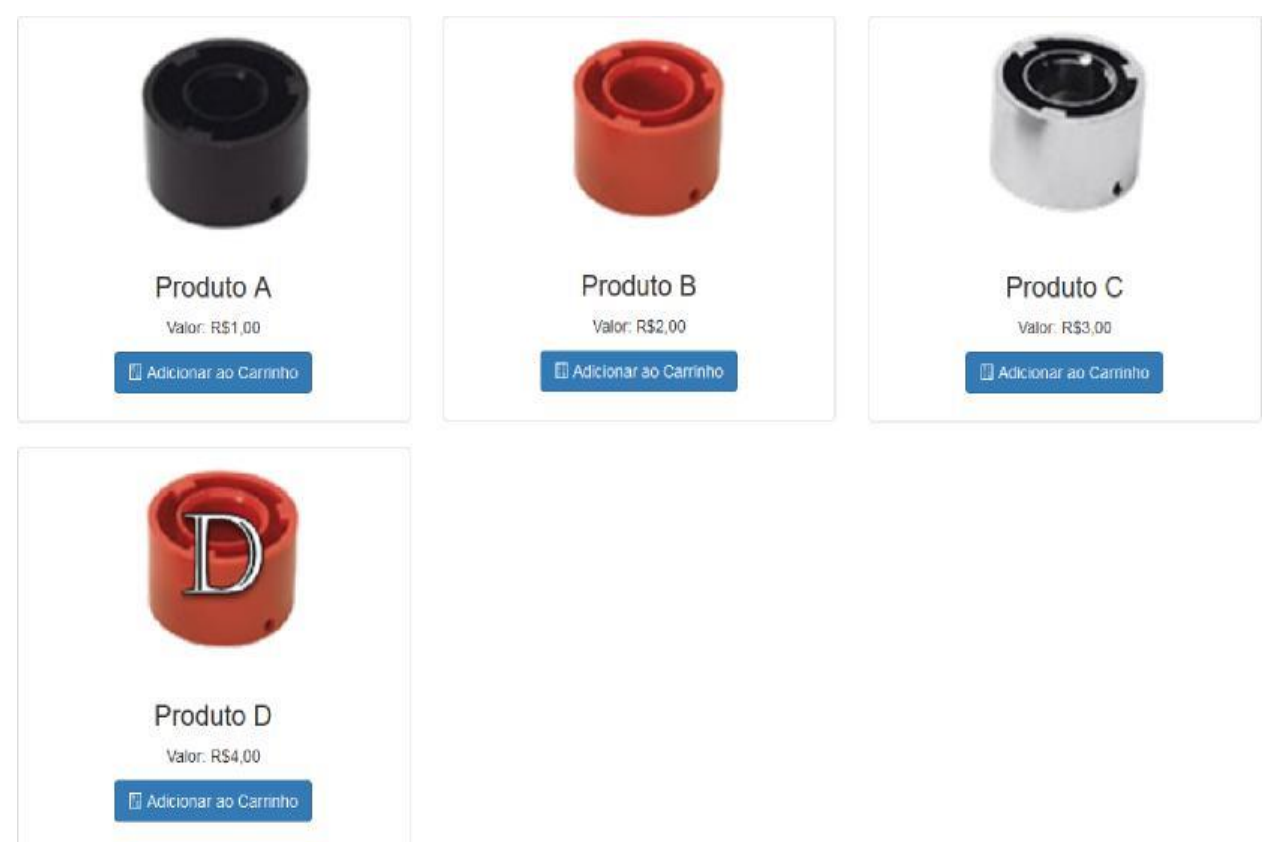

Figura 1 - Tela principal da Página Web. 


\section{Conheça nossos Produtos}
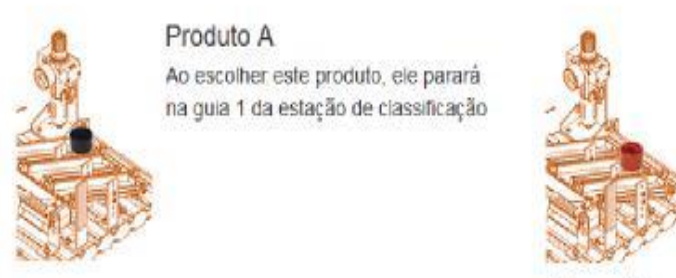

Produto B

Ao escolher este produto, ele parará

na guia 2 da estaçăo de classificação

Produto C

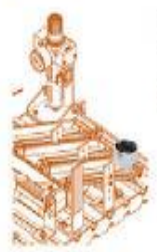

Ao escolher este procuto ele parara na guia 3 da estaçāo de classificaçāo

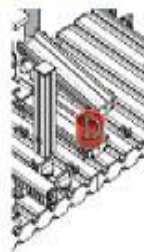

Produto D

Ao escolher este produto, ele parará

na estaçăo de teste

Figura 2 - Conhecimento dos produtos.

\section{2 SISTEMA MODULAR DE PRODUÇÃO}

Uma linha de produção de uma fábrica pode ser composta de células individuais, onde cada unidade possui uma função específica no processo, entre vários exemplos, pode-se destacar distribuição, teste, processamento, manipulação, montagem, armazenamento e classificação. Através das estações de Sistema Modular de Produção é possível gerar combinações para atingir aos requisitos de múltiplas aplicações, as quais podem representar funcionalidades de um processo real em grande escala [6]. Na seguinte figura é possível ver um exemplo de uma bancada formada por um conjunto de estações de Sistema Modular de Produção como mostra a figura 3:

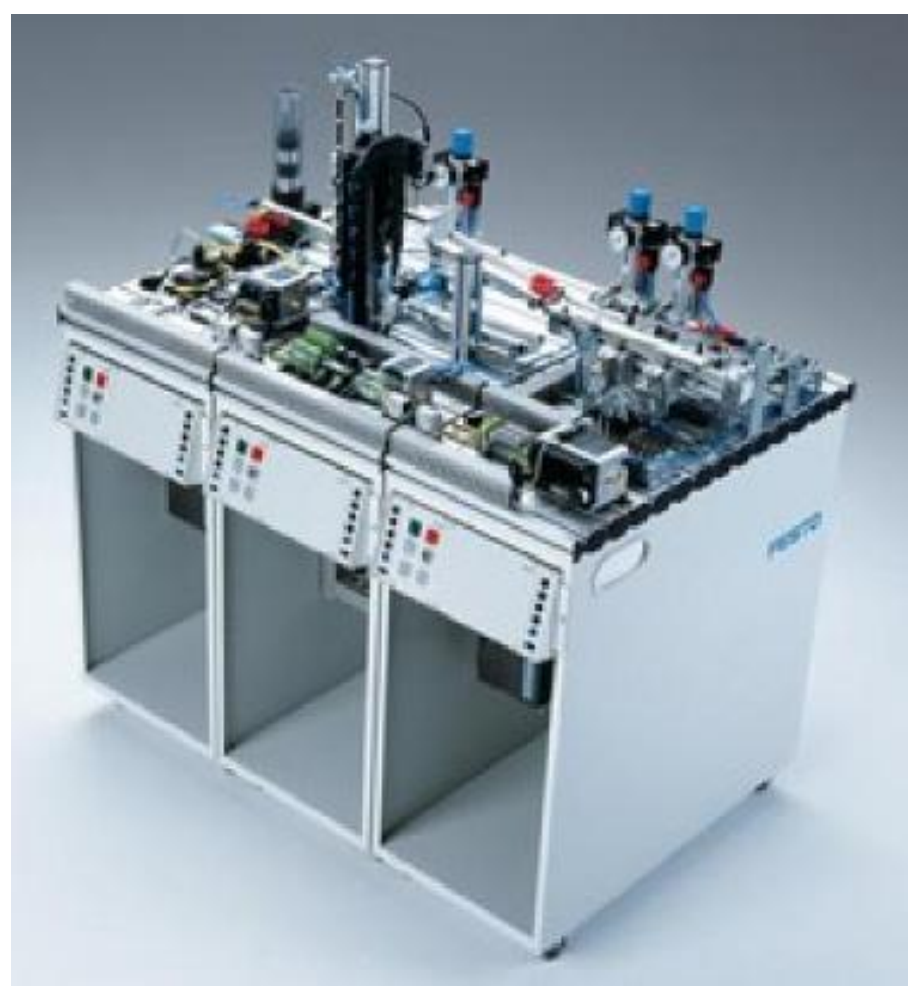

Figura 3 - Estações de Sistema Modular de Produção. 
A bancada de Sistema Modular de Produção que se encontra no laboratório de automação da Escola Superior de Tecnologia da UEA é dividida em três estações: distribuição, teste e classificação.

A estação de distribuição cumpre o papel de selecionar as peças que estão no compartimento e transferi-las à estação de teste por meio de um dispositivo com movimento rotacional utilizando uma ventosa. A estação de teste em seguida realiza sua função, que é determinar os atributos do material de uma peça, checar a altura da mesma, e então, rejeitá-la ou repassá-la para a próxima estação. A estação seguinte é a de classificação, que por último, divide as peças em três escorregadores. A figura 4 mostra a modelagem em Rede de Petri das estações de sistema modular de produção em conjunto com a aplicação na página Web:

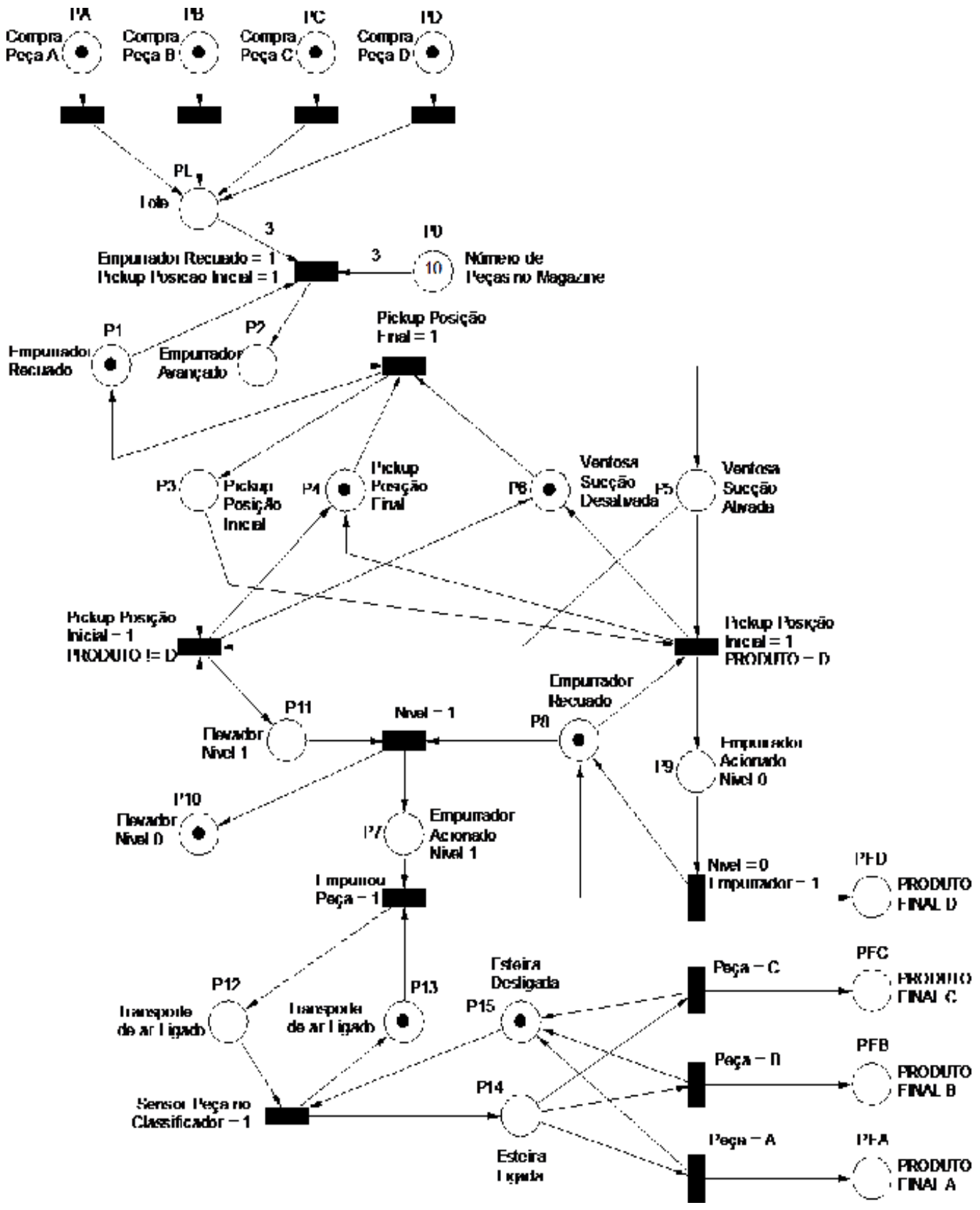

Figura 4 - Modelagem do sistema em Rede de Petri. 


\section{SISTEMA SUPERVISÓRIO}

Unindo os pensamentos de [7] e [8], pode-se dizer que o sistema supervisório se destina a capturar e armazenar em um banco de dados, informações sobre um processo de produção. Possibilitando através do acesso a esses dados, a representação de objetos estáticos e animados de forma amigável, que, juntos, representam todo o processo de uma planta em uma IHM (Interface Humano-Máquina).

Segundo [9], "com a evolução da tecnologia, foram incorporadas funções nunca antes imaginadas para um sistema supervisório. Sempre baseadas nas necessidades e requisições dos usuários finais, são implementadas ferramentas para as mais diversas finalidades". E utilizando-se deste pensamento, o sistema supervisório que será desenvolvido nesta pesquisa procura trazer inovações através da implementação de novas funcionalidades por meio da aplicação do conceito de Internet das coisas.

\section{III.1 SISTEMA SUPERVISÓRIO EM JAVA PARA A BANCADA DE SISTEMA MODULAR DE PRODUÇÃO}

Para o desenvolvimento do Sistema Supervisório foi escolhido à linguagem Java, pois, diferente de outras linguagens de programação, sua execução não está diretamente relacionada com o Sistema Operacional, pois o código é executado em uma máquina virtual chamada de JVM
(Java Virtual Machine), possibilitando assim a portabilidade do software [10]. Além disso, a linguagem Java é orientada a objetos, o que torna mais simples e rápida a realização de alterações no sistema [11].

Antes de se iniciar o desenvolvimento de um sistema, deve-se realizar o levantamento dos pré-requisitos, ou seja, devese especificar qual é a funcionalidade do software. Com a proposta de aplicação do conceito de Internet das Coisas, os requisitos do sistema supervisório foram os seguintes:

- Controle de Estoque: tem a função de cadastro e controle de matéria prima. Quando ocorre a falta de material, o pedido é feito automaticamente, sendo apenas necessário ser aprovado pelo setor de compras;

- Setor de compras: simula a função de compra de matéria prima, ao confirmar a compra, o material é acrescido ao estoque;

- Monitoramento das estações de Sistema Modular de Produção: realiza a monitoração em tempo real do funcionamento das três estações, onde é possível ver todos os produtos que se encontram em fabricação com as informações de quem comprou, em qual estação se encontra e qual será o produto final a ser produzido. Para uma visão mais completa do monitoramento, pode-se selecionar uma das estações e acompanhar o estado dos sensores e atuadores da mesma;

- Acompanhamento de Produção: possui o histórico de todos os produtos fabricados. A figura 5 mostra a tela principal de monitoramento do sistema supervisório.

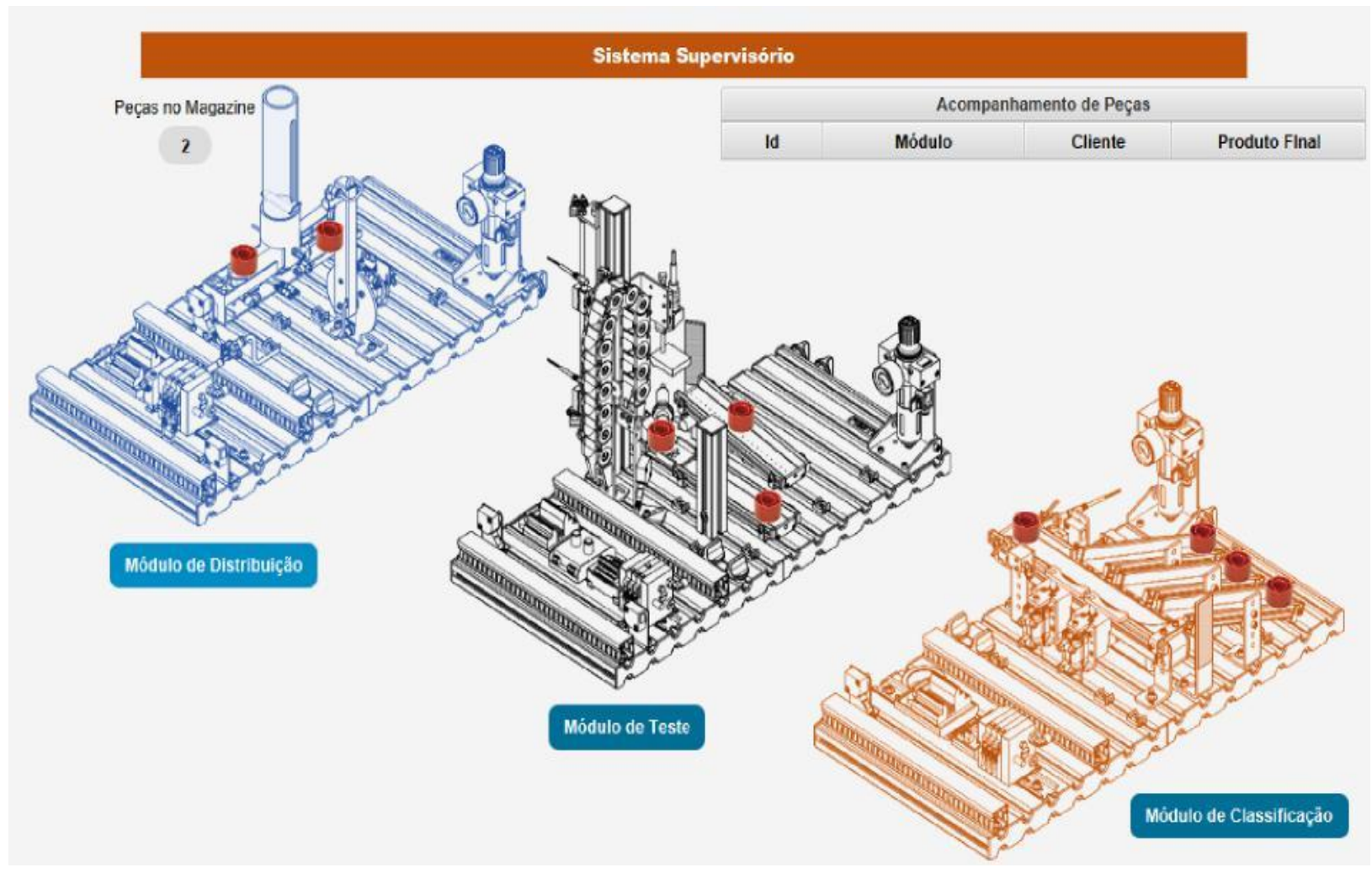

Figura 5 - Tela Principal de Monitoramento. 


\section{RESULTADOS OBTIDOS}

A figura 1 mostra a tela da página Web projetada para a simulação de compras de produtos. $\mathrm{O}$ desenvolvimento foi realizado em Java e HTML, possibilitando que a aplicação realize o que havia sido proposto. Os testes de funcionamento estão sendo executados em um servidor local, se comunicando com o mesmo banco de dados do sistema supervisório, porém é possível que a mesma página seja hospedada em um servidor web, possuindo seu próprio domínio.

A partir da modelagem em Rede de Petri, mostrada na figura 3, é possível entender o funcionamento das estações de sistema modular de produção, mostrando os eventos que ocorrem dada às circunstâncias que ocorrem no processo, denominadas transições.com base nesta modelagem, a programação dos CLP's se tornou mais simples, pois os mesmos foram programados em linguagem Ladder, que utiliza um padrão de funções lógicas que se assemelham aos expressos na modelagem.

O sistema supervisório pode realizar a comunicação direta com os dados de compras advindos da página web, e com base nesses dados, o sistema pode salvar no chip RFID de cada matéria prima, as informações de qual foi comprador e como o produto deve ser fabricado.

\section{CONCLUSÃO}

A partir dos resultados obtidos pode-se afirmar que a aplicação do conceito de Internet das Coisas permite que os sistemas se tornem mais autônomos, visto que o próprio sistema supervisório toma diversas decisões importantes para o processo.

Apesar de ter sido aplicado em escala laboratorial, foi comprovado que o sistema pode atuar em uma planta industrial, dado que as estações de sistema modular de produção têm como objetivo a representação de um processo em grande escala.

\section{REFERÊNCIAS}

[1] VENTURELLI, M. Indústria 4.0: Uma Visão da Automação Industrial. 2014. Disponível em: <http://pt.slideshare.net/MHVenturelli/industria-40$\underline{38600598>}$. Citado na página 2.

[2] CORREIA, J. et al. A internet das coisas. 2011. Disponível em: <http://ssti1-1112.wikidot. com/a-internetdas-coisas $>$. Citado na página 2.

[3] MARÃO, G. A. Internet das coisas: Oportunidades e perspectivas da nova revolução digital para o brasil. In: Seminário Internacional BNDES. [s.n.], 2014. p. 3. Disponível em: <http://www.bndes.gov.br/SiteBNDES/export/sites/default/b ndes pt/Galerias/Arquivos/ conhecimento/seminario/seminario_internet_das_coisas/Gabr iel_Marao.pdf $>$. Citado na página 2.
[4] VERMESAN, O.; FRIESS, P. Internet of Things: Converging Technologies for Smart Environments and Integrated Ecosystems. [S.1.]: River, 2013. Citado na página 2.

[5] ATZORI, L.; IERA, A.; MORABITO, G. The internet of things. In: A survey. [S.1.]: Elsevier Journal, 2010. Citado na página 2.

[6] FESTO DIDADIC. MPS: The modular production system: From module to learning factory. Denkendorf, 2006. Citado na página 4.

[7] MOTT, A. O que são sistemas supervisórios. 2012. Disponível em: <http://www. automacaoindustrial.info/o-que$\underline{\text { sao-sistemas-supervisorios/> }}$. Citado na página 5.

[8] KONDRASOVAS, I. Sistemas Supervisórios e Protocolos de Comunicação Industrial. 2013. Disponível em: <http://www.joinville.udesc.br/portal/professores/kondrasovas/ materiais/5_Sistemas_Super.pdf $>$. Citado na página 5.

[9] PAIOLA, C. E. G. O papel do supervisório no atual contexto tecnológico. InTech, v. 132, p. 8, 2012. Citado na página 5 .

[10] ROMANATO, A. Entenda como funciona a JAVA virtual machine (JVM). 2015. Disponível em: <http://www.devmedia.com.br/articles/viewcomp_forprint. asp?comp=27624> . Citado na página 5 .

[11] CAELUM. Java e Orientações a Objetos. [S.1.], 2003. Disponível em: <http://www.caelum. com.br/apostila-javaorientacao-objetos.html >. Citado na página 5 . 